\title{
NORMAL HOPF SUBALGEBRAS IN COCYCLE DEFORMATIONS OF FINITE GROUPS
}

\author{
CÉSAR GALINDO AND SONIA NATALE
}

\begin{abstract}
Let $G$ be a finite group and let $\pi: G \rightarrow G^{\prime}$ be a surjective group homomorphism. Consider the cocycle deformation $L=H^{\sigma}$ of the Hopf algebra $H=k^{G}$ of $k$-valued linear functions on $G$, with respect to some convolution invertible 2-cocycle $\sigma$. The (normal) Hopf subalgebra $k^{G^{\prime}} \subseteq k^{G}$ corresponds to a Hopf subalgebra $L^{\prime} \subseteq L$. Our main result is an explicit necessary and sufficient condition for the normality of $L^{\prime}$ in $L$.
\end{abstract}

\section{Introduction And Main Results}

Let $H$ be Hopf algebra over the field $k$. The category ${ }^{H} \mathcal{M}$ of its finite dimensional corepresentations is a tensor category. Tensor categories of this form are characterized, via tannakian reconstruction arguments, as those possessing a fiber functor with values in the category Vec of finite dimensional vector spaces over $k$. The corepresentation categories of the finite dimensional Hopf algebras $H$ and $L$ are equivalent if and only if $L=H^{\sigma}$ is a cocycle deformation of $H$ in the sense of [3], that is, $L=H$ as a coalgebra, and the multiplication in $L$ is given by

$$
h_{\cdot \sigma} g=\sigma\left(h_{1}, g_{1}\right) h_{2} g_{2} \sigma^{-1}\left(h_{3}, g_{3}\right),
$$

for some convolution invertible normalized 2-cocycle $\sigma: H \otimes H \rightarrow k$. Equivalently, the dual Hopf algebra $L^{*}$ is obtained from $H^{*}$ via a twisting of the comultiplication through $\sigma \in H^{*} \otimes H^{*}$.

A Hopf subalgebra of a finite dimensional Hopf algebra $H$ is called normal if it is invariant under the (left or right) adjoint action of $H ; H$ is called simple if it contains no proper normal Hopf subalgebra. For instance, the group algebra of finite simple group is an example of a (trivial) simple Hopf algebra.

In a previous paper [4 we gave a series of examples showing that the notions of simplicity and (semi)solvability of a (semisimple) Hopf algebra are not twist invariants; that is, they are not categorical notions. The examples in loc. cit. were obtained as twisting deformations of finite groups; namely

Date: November 21, 2007.

1991 Mathematics Subject Classification. 16W30.

This work was partially supported by CONICET, Fundación Antorchas, Agencia Córdoba Ciencia, ANPCyT and Secyt (UNC). 
we proved that there exist finite groups which are not simple, and even supersolvable in certain cases, that admit twisting deformations which are simple as Hopf algebras.

In this paper we determine necessary and sufficient conditions, in grouptheoretical terms, for a quotient of a twisting deformation of a finite group to be normal. Any twisting deformation (of the comultiplication) preserves quotient Hopf algebras. We consider here the problem of a twisting preserving normality of such a quotient. We use for this the classification of twisting in group algebras due to Movshev and Davydov [7, 2] and the results of Schauenburg on Galois correspondences [10].

Another way of regarding twisting deformations is through the language of Hopf Galois extensions. If $A$ is an $H$-Galois extension of $k$, also called an $H$ Galois object, then there exists a Hopf algebra $L$, called the left Galois Hopf algebra of $A$, such that $A$ is an $(L, H)$-bigalois object and moreover the functor $\mathcal{F}_{A}:{ }^{L} \mathcal{M} \rightarrow{ }^{H} \mathcal{M}, \mathcal{F}_{A}(V)=A \square_{H} V$, is a tensor equivalence. Conversely, if ${ }^{L} \mathcal{M}$ and ${ }^{H} \mathcal{M}$ are tensor equivalent, then there exists an $(L, H)$-bigalois object $A$ such that the equivalence is essentially $\mathcal{F}_{A}[9]$.

In this context, the Hopf algebra $L$ is a cocycle deformation of $H, L=H^{\sigma}$, and the $H$-Galois object can be identified with $A=H^{(\sigma)}$, where $H^{(\sigma)}=H$ as right $H$-comodules with multiplication $h . g=\sigma\left(h_{1}, g_{1}\right) h_{2} g_{2}, h, g \in H$.

We shall work over an algebraically closed field $k$ of characteristic zero, although most results hold as well under weaker assumptions on the characteristic.

Let $G$ be a finite group and let $\pi: G \rightarrow G^{\prime}$ be an epimorphism of groups. This corresponds to an epimorphism of Hopf algebras $k G \rightarrow k G^{\prime}$, and in turn to Hopf algebra inclusion $H^{\prime}=k^{G^{\prime}} \subseteq H=k^{G}$, which is clearly normal because $k^{G}$ is commutative. Let $F \subseteq G$ be the kernel of $\pi$; so that $F$ is a normal subgroup of $G$.

Galois objects for $k^{G}$ are classified by (conjugacy classes of) pairs $(S, \alpha)$, where $S \subseteq G$ is a subgroup and $\alpha \in H^{2}\left(S, k^{*}\right)$ is a non-degenerate 2cocycle [7, 2]. Let $A=A(G, S, \alpha)$ be the $k^{G}$-Galois object associated to this data and let $L=L\left(A, k^{G}\right)$ be the left Galois Hopf algebra. So that $L^{*}$ is a twisting deformation of $k G$. There is a bijective correspondence between Hopf subalgebras $H^{\prime} \subseteq k^{G}$ and Hopf subalgebras of $L$. We consider the Hopf subalgebra $L^{\prime} \subseteq L$ corresponding to $H^{\prime} \subseteq H$. Then $A^{\prime}={ }^{\operatorname{co} L / L\left(L^{\prime}\right)^{+} A \subseteq A \text { is }}$ a $k^{G}$-subcomodule algebra. By results of Schauenburg, $L^{\prime}$ is normal in $L$ if and only if $A^{\prime}$ is stable under the Miyashita-Ulbrich action of $k^{G}$ on $A$ [10].

For $g \in G$, and $F \subseteq G$ a subgroup, we denote by $C_{F}(g)=C_{G}(g) \cap F$ the centralizer of $g$ in $F$. Suppose $F \unlhd G$. An element $s \in S$ will be called $(\alpha, F)$-regular if $\alpha(s, t)=\alpha(t, s)$, for all $t \in C_{S}(s) \cap F$. The following theorem is proved in Section 5 .

Theorem 1.1. The Hopf subalgebra $L^{\prime} \subseteq L$ is normal if and only if $F \subseteq$ $C_{G}(s)$, for every $(\alpha, F)$-regular element $s \in S$. 
The proof of the theorem reduces to determining under what conditions the subalgebra of invariants $A^{F}$ is stable under the Miyashita-Ulbrich action of $k^{G}$, where $F$ is the kernel of the epimorphism $G \rightarrow G^{\prime}$. We find these conditions, more generally, for the case where $F$ is any (not necessarily normal) subgroup of $G$; see Theorem 5.1,

Using Theorem 1.1 we prove generalizations of some of the results in [4]; see Theorem 6.2 and Corollary 6.5. We also give an alternative proof of the simplicity of a family of twisting deformations of certain supersolvable groups in Theorem 6.6.

The paper is organized as follows: in Section 2 we give a brief account of results on Hopf Galois extensions that will be needed in the sequel. In particular, we describe in Subsection 2.2 the Galois correspondence of Schauenburg and the conditions under which normal Hopf subalgebras are preserved under this correspondence. In Section 3 we review the classification of Hopf Galois objects for finite groups due to Davydov and Movshev [2, 7]; in this context, we give a description of the Miyashita-Ulbrich action. In Section 4 we characterize the subalgebra of invariants in a Galois object $A$ under the action of a subgroup. This is applied in Section 5 to give a necessary and sufficient condition for a quotient of a twisting of a finite group to be normal. In Section [6] we apply the previous results to some families of examples.

Acknowledgement. The authors thank M. Mombelli for his interest and valuable remarks on this paper.

\section{Preliminaries on Hopf Galois extensions}

In this section we review some results on Hopf Galois extensions that we shall need later. We refer the reader to [9] for a detailed exposition on the subject.

Definition 2.1. Let $H$ be a Hopf algebra. Let also $A$ be a right $H$-comodule algebra with structure map $\rho: A \rightarrow A \otimes H, \rho(a)=a_{(0)} \otimes a_{(1)}$, and let $B:=A^{\text {co } H}$. The extension $B \subseteq A$ is called a right Hopf Galois extension, or a right $H$-Galois extension if the canonical map

$$
\text { can : } A \otimes_{B} A \rightarrow A \otimes H, \quad x \otimes y \mapsto x y_{(0)} \otimes y_{(1)},
$$

is bijective.

A right $H$-Galois extension of the base field $k$ will be called a right $H$ Galois object. Left $H$-Galois extensions and left $H$-Galois objects are defined similarly.

Definition 2.2. An $(L, H)$-bigalois object is an $(L, H)$-bicomodule algebra $A$ which is simultaneously a left $L$-Galois object and right $H$-Galois object.

Example 2.3. Let the Hopf algebra $H$ coact on itself through the comultiplication $\Delta$. Then the canonical map $H \otimes H \rightarrow H \otimes H, x \otimes y \mapsto x y_{(1)} \otimes y_{(2)}$ is bijective with inverse $a \otimes h \mapsto a S\left(h_{(1)}\right) \otimes h_{(2)}$. Since $\Delta$ is coassociative, $H$ is an $(H, H)$-bigalois object. 
More generally, let $\pi: H \rightarrow Q$ be an epimorphism of finite dimensional Hopf algebras. Then $H$ is a right $Q$-comodule algebra with structure map $\rho=(\mathrm{id} \otimes \pi) \circ \Delta$. By [8, Theorem 8.2.4 and Proposition 8.4.4] $H^{\operatorname{co} Q} \subseteq H$ is a right $Q$-Galois extension.

Assume from now on that $H$ is finite dimensional. We have a characterization of $H^{*}$-Galois objects $A$ in terms of the natural $H$-action over $A$ : $A$ is a right $H^{*}$-Galois object if and only if $A$ is finite dimensional and the map $\pi: A \# H \rightarrow$ End $A, \pi(a \# h)(b)=a(h . b)$, is an isomorphism.

Proposition 2.4. Every right $H$-Galois object is isomorphic to a crossed product $H^{(\sigma)}=k \#_{\sigma} H$, where $\sigma: H \otimes H \rightarrow k$ is an invertible 2-cocycle. Moreover, if $H$ is cosemisimple, then $H^{(\sigma)}$ is semisimple.

On the other hand, $H^{(\sigma)}$ is a right $H$-Galois object, for all such $\sigma$.

Proof. Let $A$ be a right $H$-Galois object. By [8, Proposition 8.3.6], $A \simeq$ $k \#{ }_{\sigma} H$, where $\sigma: H \otimes H \rightarrow k$ is an invertible 2-cocycle, since $A \# H^{*} \simeq$ End $A$ is simple artinian. Now by [8, Theorem 7.4.2] if $H$ is cosemisimple and finite dimensional, $A \simeq k \#_{\sigma} H$ is semisimple.

For any right $H$-Galois object $A$ there is an associated Hopf algebra $L=$ $L(A, H)$, called the left Galois Hopf algebra, such that $A$ is in a natural way an $(L, H)$-bigalois object.

In the context of Proposition 2.4 this Hopf algebra is the cocycle deformation $H^{\sigma}$ of $H$, obtained from $H$ keeping the coalgebra structure unchanged and deforming the multiplication via

$$
h_{\cdot \sigma} g=\sigma\left(h_{1}, g_{1}\right) h_{2} g_{2} \sigma^{-1}\left(h_{3}, g_{3}\right) .
$$

See [9, Proposition 3.1.6].

In the dual situation, we have that $L^{*}=\left(H^{*}\right)^{\sigma}$ is obtained from $H^{*}$ through a twisting of the comultiplication: $\Delta_{\sigma}(x)=\sigma \Delta(x) \sigma^{-1}, x \in L^{*}$.

2.1. Miyashita-Ulbrich action. Let $B \subseteq A$ be an $H$-Galois extension. The Miyashita-Ulbrich action of $H$ on the centralizer $A^{B}$ of $B$ in $A$, makes $A^{B}$ into a commutative algebra in $\mathcal{Y} \mathcal{D}_{H}^{H}$.

Definition 2.5. Let $H$ be a Hopf algebra, and $A$ an $H$-Galois extension of $B$. The Miyashita-Ulbrich action of $H$ on $A^{B}$ is defined by $x<h=h^{[1]} x h^{[2]}$, $x \in A^{B}, h \in H$, where $\operatorname{can}\left(h^{[1]} \otimes h^{[2]}\right)=1 \otimes h$.

The Miyashita-Ulbrich action of $H$ on the $H$-Galois object $A$ is characterized as the unique map $A \otimes H \rightarrow A, x \otimes h \mapsto x<h$, such that $x y=y_{(0)}\left(x \leftarrow y_{(1)}\right)$, for all $x, y \in A$.

Example 2.6. Recall that a Hopf subalgebra $H^{\prime} \subseteq H$ is called normal if it is stable under the (left or right) adjoint action of $H$.

Consider the $H$-Galois object $A=H$ as in Example 2.3. In this case, the Miyashita-Ulbrich action coincides with the right adjoint action of $H$ on itself: $x . h=\mathcal{S}\left(h_{(1)}\right) x h_{(2)}, x, h \in H$. 
Therefore $H^{\prime} \subseteq H$ is a normal Hopf subalgebra if and only if it is stable under the Miyashita-Ulbrich action. In the next subsection we recall some results of Schauenburg that we shall use later and which generalize this situation.

2.2. Galois correspondences. Let $L, H$ be finite dimensional Hopf algebras. Let $A$ be a nonzero $(L, H)$-bigalois object.

Let $\operatorname{Quot}(L)$ and $\operatorname{Sub}_{\mathrm{ff}}^{H}(A)$ be, respectively, the set of coideal left ideals $I \subseteq L$, and the set of $H$-comodule subalgebras $B \subseteq A$ such that $A$ is left faithfully flat over $B$. In view of results of Schauenburg [10, Theorem 3.6] there are mutually inverse bijections

$$
\operatorname{Quot}(L) \leftrightarrows \operatorname{Sub}_{\mathrm{ff}}^{H}(A)
$$

defined as follows: $I \in \operatorname{Quot}(L)$ is assigned to ${ }^{\text {co } L / I} A \subseteq A$, and $B \subseteq A$ goes to the coideal left ideal $\mathcal{J}(B)$ with $L / \mathcal{J}(B):=\left(A \otimes_{B} A\right)^{\text {co } H}$. We note that faithful flatness assumptions in 10 are guaranteed in the finite dimensional context by the results of Skryabin [12].

By [10, Corollary 3.13] there is a bijective correspondence between Hopf subalgebras of $H$ and $L$. Let $H^{\prime} \subseteq H$ be a Hopf subalgebra. Then $A\left(H^{\prime}\right)=$ $A \square_{H} H^{\prime} \subseteq A \square_{H} H \simeq A$ is a right $H^{\prime}$-Galois object. Let $L^{\prime}=L\left(A\left(H^{\prime}\right), H^{\prime}\right)$. Then $L^{\prime} \subseteq L$ is a Hopf subalgebra in a unique way such that $A\left(H^{\prime}\right) \subseteq A$ is an $(L, H)$-bicomodule subalgebra. In this case we have that $A\left(H^{\prime}\right)$ is an $\left(L^{\prime}, H^{\prime}\right)$-bigalois object, and $A\left(H^{\prime}\right)={ }^{\mathrm{co}} \bar{L} A, L^{\prime}={ }^{\mathrm{co}} \bar{L} L, \bar{L}=L / L\left(L^{\prime}\right)^{+}$.

Suppose $H^{\prime} \subseteq H$ is a Hopf subalgebra. The corresponding Hopf subalgebra $L^{\prime} \subseteq L$ gives rise in turn to a coideal left ideal $I=L\left(L^{\prime}\right)^{+} \in \operatorname{Quot}(L)$, which corresponds, under (2.1), to the $H$-comodule subalgebra ${ }^{\text {co } L / I} A=$ $A\left(H^{\prime}\right)$.

By [10, Theorem $3.8(\mathrm{i})], I=L\left(L^{\prime}\right)^{+} \in \operatorname{Quot}(L)$ is a Hopf ideal, that is, $L^{\prime} \subseteq L$ is a normal Hopf subalgebra, if and only if $A\left(H^{\prime}\right) \subseteq A$ is an $H$-submodule under the Miyashita-Ulbrich action. See also [11, pp. 593].

The following lemma describes the subalgebra $A\left(H^{\prime}\right)$.

Consider the quotient left $H$-module coalgebra $p: H \rightarrow H^{\prime \prime}$, where $H^{\prime \prime}=$ $H / H\left(H^{\prime}\right)^{+}$. The coalgebra $H^{\prime \prime}$ coacts on $A$ via (id $\left.\otimes p\right) \rho: A \rightarrow A \otimes H^{\prime \prime}$.

Lemma 2.7. We have $A\left(H^{\prime}\right)=\rho^{-1}\left(A \otimes H^{\prime}\right)=A^{\text {co } H^{\prime \prime}}$.

Here we identify $A\left(H^{\prime}\right)$ with a subalgebra of $A$ through the canonical isomorphism $\rho: A \rightarrow A \square_{H} H$.

Proof. It is clear that under the isomorphism $\rho: A \rightarrow A \square_{H} H, A \square_{H} H^{\prime}$ is identified with $\rho^{-1}\left(A \otimes H^{\prime}\right) \subseteq A$. The inclusion $A\left(H^{\prime}\right) \subseteq A^{\text {co } H^{\prime \prime}}$ follows since $\left.p\right|_{H^{\prime}}=\epsilon$. The other inclusion follows from the fact that $H^{\prime}=H^{\text {co } H^{\prime \prime}}$. This finishes the proof of the lemma. 
Remark 2.8. The coaction $\rho: A \rightarrow A \otimes H$ gives rise to a left action $H^{*} \otimes A \rightarrow$ $A$, such that $f . a=f\left(a_{(1)}\right) a_{(0)}$.

Consider the right coideal subalgebra $R=\left(H^{\prime \prime}\right)^{*} \subseteq H^{*}$. Then we have $A\left(H^{\prime}\right)=A^{\text {co } H^{\prime \prime}}=A^{R}$, where $A^{R}$ is the subalgebra of invariants in $A$ under the action of $R$.

\section{Galois extensions of Finite Groups}

Let $G$ be a finite group, and let $A$ be a $G$-module algebra, with the $G$ action $k G \otimes A \rightarrow A, g \otimes a \mapsto g$.a. Equivalently, $A$ is a $k^{G}$-comodule algebra with respect to the coaction $\rho: A \rightarrow A \otimes k^{G}$ defined by

$$
\rho(a)=\sum_{g \in G} g \cdot a \otimes e_{g}
$$

Here, and elsewhere in this paper, $\left(e_{g}\right)_{g \in G}$ denotes the basis of $k^{G}$ consisting of the canonical idempotents $e_{g}(h)=\delta_{g, h}, g, h \in G$.

Let $S$ be a finite group and let $\alpha \in Z^{2}\left(S, k^{*}\right)$ be a 2-cocycle. For each $s \in S$, we shall use the notation $x_{s} \in k_{\alpha} S$ to indicate the corresponding element in the twisted group algebra $k_{\alpha} S$. Thus $\left(x_{s}\right)_{s \in S}$ is a basis of $k_{\alpha} S$ and, in this basis, $x_{s} x_{t}=\alpha(s, t) x_{s t}$.

Recall that an element $s \in S$ is called $\alpha$-regular if $\alpha(s, t)=\alpha(t, s)$, for all $t \in C_{S}(s)$. This definition depends only on the class of $s$ under conjugation. The 2-cocycle $\alpha$ is called non-degenerate if and only if $\{1\}$ is the only $\alpha$ regular class in $S$.

Suppose $\alpha$ is a non-degenerate 2-cocycle on $S$ and the characteristic of $k$ is relatively prime to $|S|$. Then the twisted group algebra $k_{\alpha} S$ is naturally a $k^{S}$ Galois object with left $S$-action given by $s \triangleright x_{t}=x_{s} x_{t} x_{s}^{-1}$. This Galois object depends only on the cohomology class of $\alpha$ in $H^{2}\left(S, k^{*}\right)$ [2, Proposition 3.5]. In this case, the Miyashita-Ulbrich action $k_{\alpha} S \otimes k^{S} \rightarrow k_{\alpha} S$ corresponds to the standard $S$-grading of $k_{\alpha} S$, with respect to which $x_{t}$ is homogeneous of degree $t \in S$.

We note for future use:

Lemma 3.1. [5. Lemma 3.6] Let $G$ be a finite group. Then any $\alpha \in$ $Z^{2}\left(G, k^{*}\right)$ is cohomologous to $\beta \in Z^{2}\left(G, k^{*}\right)$ such that $\beta\left(x, x^{-1}\right)=1$, for all $x \in G$.

We may and shall assume from now on that the non-degenerate 2-cocycle $\alpha \in Z^{2}\left(S, k^{*}\right)$ satisfies $\alpha\left(s, s^{-1}\right)=1$, for all $s \in S$.

This assumption implies that, in the twisted group algebra $k_{\alpha} S$, we have $x_{t}^{-1}=x_{t^{-1}}$, for all $t \in S$.

Theorem 3.2. [2, 7]. Isomorphism classes of $k^{G}$-Galois objects are in oneto-one correspondence with conjugacy classes of pairs $(S, \alpha)$, where $S$ is a subgroup of $G$ and $\alpha \in Z^{2}\left(S, k^{*}\right)$ is a non-degenerate 2-cocycle. 
The $k^{G}$-Galois object corresponding to the pair $(S, \alpha)$ can be constructed as the algebra of $S$-invariant functions

$$
A(G, S, \alpha)=\left\{r: G \rightarrow k_{\alpha} S: \quad f(s g)=s \triangleright f(g)\right\},
$$

where $k_{\alpha} S$ is the twisted group algebra, with action $s \triangleright x_{t}=x_{s} x_{t} x_{s}^{-1}, s, t \in S$, and the $G$-action on $A$ is $(g . f)(h)=f(h g)$.

The following proposition gives an alternative description of the Galois object $A(G, S, \alpha)$ that will be convenient to our purposes.

Proposition 3.3. There is an isomorphism of G-algebras

$$
A(G, S, \alpha) \simeq \operatorname{Ind}_{S}^{G} k_{\alpha} S=k G \otimes_{k S} k_{\alpha} S,
$$

where the left $G$-action and the multiplication are given, respectively, by

$$
\begin{aligned}
g^{\prime} . g \otimes x & =g^{\prime} g \otimes x, \\
(g \otimes x)(h \otimes y) & = \begin{cases}0, & h^{-1} g \notin S, \\
h \otimes\left(h^{-1} g . x\right) y, & h^{-1} g \in S .\end{cases}
\end{aligned}
$$

Proof. Let $A=A(G, S, \alpha)$. By definition, $A=\operatorname{Hom}_{k S}\left(k G, k_{\alpha} S\right)$. Using the version of Frobenius reciprocity in [1, Proposition (10.21)], we have isomorphisms $\operatorname{Hom}_{k S}\left(k G, k_{\alpha} S\right) \simeq \operatorname{Hom}_{k G}\left(k G, k G \otimes_{k S} k_{\alpha} S\right) \simeq k G \otimes_{k S} k_{\alpha} S$.

Write $G=\cup_{i=1}^{r} g_{i} S$, where $g_{i}, 1 \leq i \leq r$, is a set of representatives of the left cosets of $S$ in $G$. An isomorphism $\phi: A(G, S, \alpha) \rightarrow k G \otimes_{k S} k_{\alpha} S$ is defined explicitly by

$$
\phi(f)=\sum_{i} g_{i} \otimes f\left(g_{i}^{-1}\right) .
$$

Its inverse $\psi: k G \otimes_{k S} k_{\alpha} S \rightarrow A(G, S, \alpha)$ is determined by

$$
\psi(g \otimes x)(b)=\lambda(b g) x,
$$

where $\lambda: G \rightarrow k S$ is the map $\lambda(g)=g$, if $g \in S$, and $\lambda(g)=0$, otherwise. Of course, these isomorphisms do not depend on the choice of left coset representatives. Moreover, they transform the $G$-algebra structure on $A$ into the $G$-algebra structure on $k G \otimes_{k S} k_{\alpha} S$ given by formulas (3.1) and (3.2). This proves the proposition.

From now on we shall use the identification $A=A(G, S, \alpha) \simeq k G \otimes_{k S} k_{\alpha} S$ given in Proposition 3.3 .

We next describe the Miyashita-Ulbrich action $\leftarrow: A \otimes k^{G} \rightarrow A$ in terms of this identification. First note that, in our context, this action corresponds to a $G$-grading on $A$ : $A=\oplus_{g \in G} A_{g}$, such that $A_{g}=A<e_{g}, g \in G$. We shall call it the Miyashita-Ulbrich grading.

Lemma 3.4. The Miyashita-Ulbrich grading on $A$ is determined by

$$
\left|g \otimes x_{s}\right|:=g s g^{-1}
$$

for all $g \in G, s \in S$. 
Proof. Recall that the Miyashita-Ulbrich action of a Hopf algebra $H$ over the $H$-Galois object $A$ is characterized as the unique map $A \otimes H \rightarrow A$, $x \otimes h \mapsto x<h$, such that $x y=y_{(0)}\left(x \leftarrow y_{(1)}\right)$, for all $x, y \in A$. Thus, for $H=k^{G}$, we have $A_{g}=\{a \in A \mid a x=(g \cdot x) a$, for all $x \in A\}$.

Let $g, h \in G, s, t \in S$. We have

$$
\begin{aligned}
\left(\left(g s g^{-1}\right) \cdot\left(h \otimes x_{t}\right)\right)\left(g \otimes x_{s}\right) & =\left(g s g^{-1} h \otimes x_{t}\right)\left(g \otimes x_{s}\right) \\
& =\left\{\begin{array}{l}
0, \quad s g^{-1} h \notin S, \\
g \otimes\left(s g^{-1} h . x_{t}\right) x_{s}, \quad s g^{-1} h \in S .
\end{array}\right.
\end{aligned}
$$

Clearly, $s g^{-1} h \in S$ if and only if $h^{-1} g \in S$ and, in this case,

$$
g \otimes\left(s g^{-1} h . x_{t}\right) x_{s}=g \otimes x_{s}\left(g^{-1} h . x_{t}\right)=h \otimes\left(h^{-1} g . x_{s}\right) x_{t},
$$

the first equality because $x_{s}$ is homogeneous of degree $s$ with respect to the Miyashita-Ulbrich $S$-grading in $k_{\alpha} S$. Hence

$$
\left(\left(g s g^{-1}\right) \cdot\left(h \otimes x_{t}\right)\right)\left(g \otimes x_{s}\right)=\left(g \otimes x_{s}\right)\left(h \otimes x_{t}\right) .
$$

Since this holds for all $g, h \in G, s, t \in S$, then $g \otimes x_{s}$ is homogeneous of degree $g s g^{-1}$, as claimed. This finishes the proof of the lemma.

\section{The subalgebra $A^{F}$ of $F$-invariants for a subgroup $F$}

Following [5, Chapter 7, Section 9] we shall consider monomial representations in order to describe a basis of the subalgebra of invariants $A^{F}$.

Let $F$ be a finite group. A monomial space over $k$ consists of the data $\left(V, X,\left(V_{x}\right)_{x \in X}\right)$, where $V$ is a vector space, $X$ is finite set, and $\left(V_{x}\right)_{x \in X}$ is a family of one-dimensional subspaces of $V$ indexed by $X$, such that $V=\oplus_{x \in X} V_{x}$.

By a monomial representation of a group $F$ on $\left(V, X,\left(V_{x}\right)_{x \in X}\right)$ we mean a group homomorphism

$$
\Gamma: F \rightarrow G L(V),
$$

such that for each $\sigma \in F, \Gamma(\sigma)$ permutes the $V_{x}$ 's; hence the action on $V$ induces an action by permutations of $F$ on $X$.

For each $x \in X$, we shall denote $F(x)$ the stabilizer of $x$. We say that an element $x \in X$ is regular under the monomial action of $F$ if, for all $\sigma \in F(x)$, $\Gamma(\sigma)$ is the identity map on $V_{x}$.

Lemma 4.1. [5, Lemma 9.1]. Let $\left(V, X,\left(V_{x}\right)_{x \in X}\right)$ be a monomial space, and let $\Gamma: F \rightarrow G L(V)$ a monomial representation of $F$ on $\left(V, X,\left(V_{x}\right)_{x \in X}\right)$.

(1) If $x \in X$ is a regular element under the monomial action of $F$, then so are all elements in the $F$-orbit of $x$.

(2) Let $T$ be a set of representatives of the regular orbits of $X$. For each $t \in T$, let $w_{t}$ be a nonzero vector of $V_{t}$. Set

$$
v_{t}=\sum_{\sigma \in Y_{t}} \Gamma(\sigma) w_{t}
$$


where $Y_{t}$ is a set of left coset representatives for $F(t)$ in $F$. Then $\left(v_{t}\right)_{t \in T}$ is a basis of the subspace $V^{F}$ of $F$-invariants in $V$.

Remark 4.2. The dimension of $V^{F}$ is equal to the number of regular $F$-orbits under the monomial action of $F$ on $X$.

Note in addition that the set $\left\{\Gamma(\sigma) w_{t}\right\}_{t \in T, \sigma \in Y_{t}}$ is linearly independent in $V$. Indeed, $\Gamma(\sigma) w_{t}$ belongs to $V_{\sigma . t}$. Because elements in $T$ are not conjugated under the action of $F$, and $\sigma$ runs over a set of left coset representatives of $F(t)$, all these elements have pairwise distinct degree of homogeneity and are thus linearly independent.

Let $I=\left\{g_{1}, g_{2}, \ldots, g_{n}\right\}$ be a set of representatives of the right cosets of $S$ in $G$, so that $\left\{g_{i} \otimes_{k S} x_{s}\right\}_{s \in S, i \in I}$ is a basis of $k G \otimes_{k S} k_{\alpha} S$. It is clear that $\left(A, I \times S, k\left(g \otimes x_{s}\right)\right)$ is a monomial space.

The set $I$ carries a natural permutation action of $G$ given by $g \cdot g_{i}=g_{j}$, if and only if

$$
g g_{i}=g_{j} t, \quad t \in S .
$$

The action of $G$ on $A$ in terms of the basis $\left\{g_{i} \otimes_{k S} x_{s}\right\}_{s \in S, i \in I}$, has the form

$g \cdot\left(g_{i} \otimes_{k S} x_{s}\right)=g g_{i} \otimes_{k S} x_{s}=\left(g . g_{i}\right) t \otimes_{k S} x_{s}=\alpha(t, s) \alpha\left(t s, t^{-1}\right)\left(g \cdot g_{i}\right) \otimes_{k S} x_{t s t^{-1}}$.

Then, for each $g \in G$, the action of $g$ permutes the spaces $k\left(g_{i} \otimes_{k S} x_{s}\right)$; in other words, $\left(A, I \times S, k\left(g_{i} \otimes x_{s}\right)\right)$ is a monomial representation of $G$.

The induced action of $G$ on $I \times S$ is given by

$$
g \cdot\left(g_{i}, s\right)=\left(g_{j}, t s t^{-1}\right)=\left(g \cdot g_{i}, t s t^{-1}\right),
$$

with $g_{j} \in I, t \in S$, as above.

Let $F \subseteq G$ be a subgroup. In what follows we shall consider the monomial representation of $F$ on $A$ obtained by restriction.

We can regard $S$ as a left $S$-set and $G$ as a right $S$-set, with actions given by conjugation and right multiplication, respectively; that is, s.t $=s t s^{-1}$, $g . s=g s$ for $s, t \in S, g \in G$. Then we can consider the quotient set $G \times{ }_{S} S$ under the equivalence relation: $(g, s) \sim\left(g^{\prime}, s^{\prime}\right)$, if and only if, there exist $t \in S$ such that $\left(g^{\prime}, s^{\prime}\right)=\left(g . t^{-1}, t . s\right)=\left(g t^{-1}, t s t^{-1}\right)$.

The set $G \times{ }_{S} S$ is equipped with a left $G$-action given by left multiplication on the first component.

Lemma 4.3. The map $I \times S \rightarrow G \times{ }_{S} S$ that carries the element $\left(g_{i}, s\right)$ to the class of the pair $\left(g_{i}, s\right)$, is an isomorphism of $G$-sets.

Proof. For every $g \in G$, write $g=g_{i} s^{\prime}$, uniquely, for some $g_{i} \in I, s^{\prime} \in S$. Map the class of the pair $(g, s)$ to the pair $\left(g_{i}, s^{\prime} s s^{-1}\right) \in I \times S$. This gives a well defined inverse of the map in the lemma. It is clear that both maps are maps of $G$-sets.

We can identify the monomial representation $\left(A, I \times S, k\left(g_{i} \otimes x_{s}\right)\right)$ of $F$ with $\left(A, G \times \times_{S} S, k\left(g \otimes x_{s}\right)\right)$. 
Definition 4.4. We shall say that a class $(g, s) \in G \times{ }_{S} S$ is $(\alpha, F)$-regular if $\alpha(s, t)=\alpha(t, s)$, for all $t \in C_{S}(s) \cap g^{-1} F g$.

Suppose that $F$ is normal in $G$. We shall say that $s \in S$ is $(\alpha, F)$-regular if $\alpha(s, t)=\alpha(t, s)$, for all $t \in C_{S}(s) \cap F$.

Remark 4.5. In view of Lemma 4.6 below, the notion of $(\alpha, F)$-regularity is well defined, that is, it depends only on the class of $(g, s) \in G \times{ }_{S} S$.

Moreover, under the assumption that $F$ is normal in $G$, the definition of an $(\alpha, F)$-regular element $s \in S$ depends only on the conjugacy class of $s$ in $S$. In this case, for all $g \in G$, the class $(g, s)$ is $(\alpha, F)$-regular in $G \times{ }_{S} S$ if and only if $s \in S$ is $(\alpha, F)$-regular.

Lemma 4.6. The class $(g, s) \in G \times_{S} S$ is regular under the action of $F$ if and only if it is $(\alpha, F)$-regular.

Proof. The stabilizer of $(g, s)$ in $F$ is the subgroup $F(g, s)=g C_{S}(s) g^{-1} \cap F$. Let $r \in g C_{S}(s) g^{-1} \cap F, r=g t g^{-1}, t \in C_{S}(s)$. Then

$$
\begin{aligned}
g t g^{-1} \triangleright g \otimes_{k S} x_{s} & =\alpha(t, s) \alpha\left(t s, t^{-1}\right) g \otimes_{k S} x_{s} \\
& =\alpha^{-1}(s, t) \alpha(t, s) g \otimes_{k S} x_{s} \\
& =\alpha^{-1}\left(s, g^{-1} r g\right) \alpha\left(g^{-1} r g, s\right) g \otimes_{k S} x_{s},
\end{aligned}
$$

where the second equality follows because $\alpha\left(g, g^{-1}\right)=1$, for all $g \in G$. Then the class $(g, s)$ is regular under the action of $F$ if and only if $\alpha^{-1}\left(s, g^{-1} r g\right)$ $\alpha\left(g^{-1} r g, s\right)=1$, for all $r \in g^{-1} C_{S}(s) g \cap F$, i.e., if and only if $(g, s)$ is $(\alpha, F)$-regular.

Proposition 4.7. Let $T$ be a set of representatives of the regular $F$-orbits of $G \times{ }_{S} S$. For each $(g, t) \in T$, set

$$
v_{(g, t)}=\sum_{h \in Y_{(g, t)}} h g \otimes_{k S} x_{t}, \quad(g, t) \in T,
$$

where $Y_{(g, t)}$ is a set of left coset representatives of $g C_{S}(s) g^{-1} \cap F$ in $F$. Then $\left(v_{(g, t)}\right)_{(g, t) \in T}$ is a basis of the subalgebra $A^{F}$ of $F$-invariants of $A$.

Proof. We have that $\left(A, G \times_{S} S, k\left(g \otimes_{k S} x_{s}\right)\right)$ is a monomial representation of $F$. By Lemma 4.6, the stabilizers are the subgroups $g C_{S}(s) g^{-1} \cap F$. By Lemma 4.1, $\left(v_{(g, t)}\right)_{(g, t) \in T}$ is basis of $A^{F}$.

Remark 4.8. Since the $k^{G}$-Galois object $A(G, S, \alpha)$ is isomorphic to a crossed product over $k^{G}$, it is isomorphic to the regular representation $k G$ as a $G$ module. Therefore $\operatorname{dim} A(G, S, \alpha)^{F}=[G: F]$.

In view of Proposition 4.7, for all nondegenerate cocycle $\alpha$, the number of $(\alpha, F)$-regular orbits in $G \times_{S} S$ equals $[G: F]$.

\section{Normal Hopf subalgebras in COCyCle Deformations}

Let $G$ be a finite group, let $A=A(G, S, \alpha)$ be a $k^{G}$-Galois object, and consider the twisted Hopf algebra deformation $L=L\left(A, k^{G}\right)$ of $k^{G}$. 
Let $\pi: G \rightarrow G^{\prime}$ be an epimorphism of groups. This corresponds to an epimorphism of Hopf algebras $k G \rightarrow k G^{\prime}$. Let $F \subseteq G$ be the kernel of $\pi$; so that $F$ is a normal subgroup of $G$.

Dualizing the epimorphism $\pi: G \rightarrow G^{\prime}$ we obtain a Hopf algebra inclusion $H^{\prime}=k^{G^{\prime}} \subseteq H=k^{G}$, which is of course normal since $k^{G}$ is commutative.

We determine necessary and sufficient conditions, in group-theoretical terms, for the Hopf subalgebra $L^{\prime} \subseteq L$, corresponding to $H^{\prime} \subseteq H$, to be normal in $L$.

First, for any subgroup $F$ of $G$, we have:

Theorem 5.1. $A^{F}$ is a $k^{G}$-submodule of $A$ with respect to the MiyashitaUlbrich action if and only if for all $(\alpha, F)$-regular $(g, s) \in G \times{ }_{S} S, g^{-1} F g \subseteq$ $C_{G}(s)$.

Proof. We keep the notations in Proposition 4.7. Note that the condition $g^{-1} F g \subseteq C_{G}(s)$ is equivalent to $C_{F}\left(g s g^{-1}\right)=F$.

Suppose first that $C_{F}\left(g s g^{-1}\right)=F$, for all $(g, s) \in T$. Let $\sigma \in G$. By Lemma 3.4, we have

$$
\begin{aligned}
v_{(g, s)} \leftarrow e_{\sigma} & =\sum_{h \in Y_{(g, s)}} h g \otimes_{k S} x_{s} \leftarrow e_{\sigma} \\
& =\sum_{h \in Y_{(g, s)}} \delta_{\sigma,(h g) s(h g)^{-1}} h g \otimes_{k S} x_{s} \\
& =\delta_{\sigma, g s g^{-1}} v_{(g, s)} .
\end{aligned}
$$

Since this holds for all $\sigma \in G$, then $A^{F}$ is a $k^{G}$-submodule with respect to the Miyashita-Ulbrich action.

Conversely, suppose that $A^{F}$ is a $k^{G}$-submodule under the MiyashitaUlbrich action. Let $\left\{v_{\left(g_{0}, t_{0}\right)}, v_{\left(g_{1}, t_{1}\right)}, \ldots, v_{\left(g_{r}, t_{r}\right)}\right\}$ be a basis of $A^{F}$ given by Proposition 4.7

Recall that $\left\{h g_{i} \otimes_{k S} x_{t_{i}}\right\}_{h \in Y_{\left(g_{i}, t_{i}\right)},\left(g_{i}, t_{i}\right) \in T}$ is a set of linearly independent vectors in $A$; c.f. Remark 4.2 ,

We have $v_{\left(g_{0}, t_{0}\right)}=\sum_{i=1}^{k} v_{\left(g_{0}, t_{0}\right)}^{\sigma_{i}}$, where $v_{\left(g_{0}, t_{0}\right)}^{\sigma_{i}}$ is the homogeneous component of degree $\sigma_{i} \in G$ with the respect to the Miyashita-Ulbrich grading:

$$
v_{\left(g_{0}, t_{0}\right)}^{\sigma_{i}}=\sum_{h \in Y_{\left(g_{0}, t_{0}\right)}, h g_{0} t_{0} g_{0}^{-1} h^{-1}=\sigma_{i}} h g_{0} \otimes_{k S} x_{t_{0}} .
$$

Since, by assumption, $A^{F}$ is stable under the Miyashita-Ulbrich action of $k^{G}$, each one of the homogeneous components $v_{\left(g_{0}, t_{0}\right)}^{\sigma_{i}}$ is in $A^{F}$.

Thus, if $v_{\left(g_{0}, t_{0}\right)}^{\sigma_{1}}, \ldots, v_{\left(g_{0}, t_{0}\right)}^{\sigma_{l}}$ are the nonzero homogeneous components of $v_{\left(g_{0}, t_{0}\right)}$, then

$$
\left\{v_{\left(g_{0}, t_{0}\right)}^{\sigma_{1}}, \ldots, v_{\left(g_{0}, t_{0}\right)}^{\sigma_{l}}, v_{\left(g_{1}, t_{1}\right)}, \ldots, v_{\left(g_{r}, t_{r}\right)}\right\}
$$


is a set of $l+r$ linearly independent vectors in $A^{F}$. But $v_{\left(g_{0}, t_{0}\right)}, v_{\left(g_{1}, t_{1}\right)}$, $\ldots, v_{\left(g_{r}, t_{r}\right)}$ form a basis of $A^{F}$. Therefore $l=1$. Hence $v_{\left(g_{0}, t_{0}\right)}^{\sigma_{1}}=v_{\left(g_{0}, t_{0}\right)}$, and $v_{\left(g_{0}, t_{0}\right)}^{\sigma_{i}}=0$, for all $i>1$. This implies that $v_{(g, s)}$ is homogeneous for all $(\alpha, F)$-regular class $(g, s)$.

Let $(g, s)$ be an $(\alpha, F)$-regular class. We have $v_{(g, s)}<e_{u}=v_{(g, s)}$, for some $u \in G$. Thus

$$
v_{(g, s)}=\sum_{h \in Y_{(g, s)}} h g \otimes_{k S} x_{s} \leftarrow e_{u}=\sum_{h \in Y_{(g, s)}} \delta_{u,(h g) s(h g)^{-1}} h g \otimes_{k S} x_{s} .
$$

We may assume that $e \in Y_{(g, s)}$, where $e \in F$ is the identity element. Then, by linear independence of the set $\left\{h g \otimes_{k S} x_{t}\right\}_{h \in Y_{(g, t)},(g, t) \in T}$, we obtain $g s g^{-1}=u$, and also $g s g^{-1}=h g s g^{-1} h^{-1}$, for all $h \in Y_{(g, s)}$. But $(g, s)$ is an arbitrary element in the $(\alpha, F)$-regular $F$-orbit and $Y_{(g, s)}$ is any set of left coset representatives for $g C_{S}(s) g^{-1} \cap F$ in $F$. Then $g s g^{-1}=h g s g^{-1} h^{-1}$ for all $h \in F$. That is, $F=C_{F}\left(g s g^{-1}\right)$. This finishes the proof of the theorem.

As an application of Theorem 5.1 we give a proof of our main result.

Proof of Theorem 1.1. By Lemma 2.7 and the results in 2.1, it is enough to determine the conditions under which the subalgebra of invariants $A^{F}$ is stable under the Miyashita-Ulbrich action. This is a special case of Theorem 5.1. for the case when $F$ is a normal subgroup of $G$.

\section{EXAMPLES}

In this section $G$ will be a finite group, $S \subseteq G$ will be a subgroup, and $\alpha \in Z^{2}\left(S, k^{*}\right)$ a non-degenerate 2-cocycle. As before, $A=A(G, S, \alpha)$ will denote the corresponding $k^{G}$-Galois object.

We shall denote by $J \in k S \otimes k S \subseteq k G \otimes k G$ the twist corresponding to $A$. So that $(k G)^{J} \simeq L^{*}$, where $L=L(A, H)$.

As a first application of Theorem 1.1 we state the following lemma. Its interpretation in terms of twists is well-known; see [6, Lemma 5.4.1].

Lemma 6.1. Let $F$ be a normal subgroup of $G$. If $S \subseteq F$, then $A^{F}$ is a $k^{G}$-submodule of $A$ with respect to the Miyashita-Ulbrich action.

In other words, if $J \subseteq k F \otimes k F$, then $(k F)^{J} \subseteq(k G)^{J}$ is a normal Hopf subalgebra.

Proof. Let $(g, s)$ be an $(\alpha, F)$-regular class. Then $\alpha(s, t)=\alpha(t, s)$, for all $t \in C_{S}(s) \cap g^{-1} F g=C_{S}(s)$. Since $\alpha$ is non-degenerate, then $s=1$. By Theorem [5.1, $A^{F}$ is a $k^{G}$-submodule of $A$ with respect to the MiyashitaUlbrich action.

The following Theorem gives a generalization of [4, Theorem 3.3] to the case where $S$ is not necessarily abelian. 
Theorem 6.2. Suppose that $Z(G)=1$. Let $F$ be a normal subgroup of $G$, such that $[G: F]$ is prime. Then $A^{F}$ is a $k^{G}$-submodule of $A$ with respect to the Miyashita-Ulbrich action if and only if $S \subseteq F$.

In other words, the corresponding quotient $(k G)^{J} \rightarrow k(G / F)^{\bar{J}}$ is conormal, that is, dual to a normal inclusion, if and only if $J \in k F \otimes k F$.

Proof. Suppose $S \subseteq F$. Then, by Lemma 6.1, $A^{F}$ is a $k^{G}$-submodule of $A$ with respect to the Miyashita-Ulbrich action.

Conversely, suppose that $A^{F}$ is a $k^{G}$-submodule of $A$ with respect to the Miyashita-Ulbrich action. Let $s \in S$ be an $(\alpha, F)$-regular element. Then $F \subseteq C_{G}(s)$.

If $F \varsubsetneqq C_{G}(s)$, then $C_{G}(s)=G$, because $[G: F]$ is prime. Since $Z(G)=1$, we have $s=1$. If $F=C_{G}(s)$, then $\alpha(s, t)=\alpha(t, s)$, for all $t \in C_{S}(s) \cap F=$ $C_{S}(s)$. Since $\alpha$ is non-degenerate, then $s=1$ as well.

Therefore every $(\alpha, F)$-regular class is of the form $(g, 1)$. Recall that $\operatorname{dim} A^{F}$ is equal to the number of $(\alpha, F)$-regular orbits in $G \times_{S} S$. Note that $(g, 1) \sim(\sigma, 1)$ if and only if $g \in \sigma S$. Then $(g, 1)$ is conjugate to $(\sigma, 1)$ under the action of $F$ if and only if $g \in F \sigma S=\sigma F S$. Hence the number of $(\alpha, F)$-regular orbits is $[G: F S]$.

On the other hand, the dimension of $A^{F}$ is $[G: F]$, see Remark 4.8. Thus $[G: F S]=[G: F]$, and therefore $S \subseteq F$. This finishes the proof of the theorem.

6.1. Simple deformations of a family of non-solvable groups. Let $F$ be a finite simple non-Abelian group, and let $x \in F$ be an element of prime order $p$. Let $G=F \rtimes \mathbb{Z}_{p}$ be the semidirect product with respect to the action given by conjugation by $x$. Note that $Z(G)=1$.

Proposition 6.3. Let $G=F \rtimes \mathbb{Z}_{p}$, then $(k G)^{J}$ is a simple Hopf algebra if and only if $S \nsubseteq F$.

Proof. The only non-trivial normal subgroup of $G$ is $F$. By Theorem 6.2, the quotient $(k G)^{J} \rightarrow k \mathbb{Z}_{p}$ is conormal if and only if $S \subseteq F$.

Remark 6.4. For the subgroup $S=\langle x\rangle \rtimes \mathbb{Z}_{p}$, we have $S \simeq \mathbb{Z}_{p} \times \mathbb{Z}_{p}$. In particular, every $1 \neq \alpha \in H^{2}\left(S, k^{*}\right) \simeq \mathbb{Z}_{p} \times \mathbb{Z}_{p}$ is non-degenerate. That is, the Hopf algebra $k^{G}$ has at least $p-1$ non-trivial Galois objects $A(G, S, \alpha)$ for which $S \nsubseteq F$. All the corresponding twisting deformations are simple in view of Proposition 6.3.

As a consequence we get the following generalization of the results in 4, Section 3] for the symmetric groups $\mathbb{S}_{n}$.

Corollary 6.5. Let $n \geq 5$ and let $G=\mathbb{S}_{n}, F=\mathbb{A}_{n}$ be the symmetric and alternating groups on $n$ letters, respectively. For every subgroup $S \subseteq \mathbb{S}_{n}$ and non-degenerate 2-cocycle $\alpha$ on $S$, the associated deformation $\left(k \mathbb{S}_{n}\right)^{J}$ is simple if and only if $S \nsubseteq \mathbb{A}_{n}$. 
Proof. We have $G=\mathbb{A}_{n} \rtimes \mathbb{Z}_{2}=\mathbb{S}_{n}$. The statement follows from 6.3.

6.2. Example of family of super solvable groups. As a further application of Theorem 1.1 we give in this Subsection an alternative proof of the main result in [4, Section 4] (note that we drop here the assumption on the characteristic of $k$ made in loc. cit.). Let $p, q$ and $r$ be prime numbers such that $q$ divides $p-1$ and $r-1$. Let $G_{1}=\mathbb{Z}_{p} \rtimes \mathbb{Z}_{q}$ and $G_{2}=\mathbb{Z}_{r} \rtimes \mathbb{Z}_{q}$ be the only nonabelian groups of orders $p q$ and $r q$, respectively. Let $G=G_{1} \times G_{2}$ and let $\mathbb{Z}_{q} \times \mathbb{Z}_{q} \simeq S \subseteq G$ a subgroup of order $q^{2}$. In particular, $G$ is supersolvable and $Z(G)=1$.

Theorem 6.6. Let $1 \neq \alpha \in H^{2}\left(S, k^{*}\right)$. Then $(k G)^{J}$ is a simple Hopf algebra.

Proof. Note that the groups $G_{i}$ have only one normal subgroup. The subgroup $S$ is conjugated to a subgroup of the form $S=S_{1} \times S_{2}$, where $S_{i} \subseteq G_{i}$, $S_{i} \simeq \mathbb{Z}_{q}$, and if $s=\left(s_{1}, s_{2}\right) \in S$, then $C_{G}(s)=C_{G_{1}}\left(s_{1}\right) \times C_{G_{2}}\left(s_{2}\right)$.

Consider a normal subgroup $N$ of $G$ such that $A^{N}$ is a $k^{G}$-submodule of $A$ with respect to the Miyashita-Ulbrich action.

Let $s \in S$ be an $(\alpha, N)$-regular element; so that $N \subseteq C_{G}(s)$ and $\alpha(s, t)=$ $\alpha(t, s)$ for all $t \in C_{G}(s) \cap N$.

If $s_{1} \neq 1$ and $s_{2} \neq 1$ then $C_{G}(s)=S$. But $S$ contains no normal subgroups of $G$, hence $s_{1}=1$ or $s_{2}=1$. Suppose that $s_{1}=1$ and $s_{2} \neq 1$, then $C_{G}(s)=S_{1} \times G_{2} \supseteq N$, so $\alpha(s, t)=\alpha(t, s)$ for all $t \in S$. But $\alpha$ is nondegenerate, so $s=1$, which is a contradiction.

Then every $(\alpha, F)$-regular class is of the form $(g, 1)$. The same argument of the proof of Theorem 6.2 shows that $[G: N S]=[G: N]$, and thus $S \subseteq N$. This implies that $N=G$. Hence $(k G)^{J}$ contains no proper normal Hopf subalgebra. This proves the theorem.

\section{REFERENCES}

[1] C. Curtis and I. Reiner, Methods of representation theory, I, Wiley Interscience Publications, New York (1990).

[2] A. A. Davydov, Galois Algebras and Monoidal Functors between Categories of Representations of Finite Groups, J. Algebra 244 (2001), 273-301.

[3] Y. DoI, Braided bialgebras and quadratic bialgebras, Commun. Algebra 21 (1993), 1731-1749.

[4] C. Galindo and S. Natale, Simple Hopf algebras and deformations of finite groups, to appear in Math. Res. Lett. Preprint arXiv:math/0608734v2.

[5] G. Karpilovsky, Proyective Representation of Finite Groups, Pure and Applied Mathematics 94, Marcel Dekker, New York-Basel (1985).

[6] S. Natale, Semisolvability of semisimple Hopf algebras of low dimension, Memoirs Amer. Math. Soc. 186 (2007).

[7] M. Movshev, Twisting in group algebras of finite groups, Func. Anal. Appl. 27 (1994), $240-244$.

[8] S. Montgomery, Hopf Algebras and Their Action on Rings, CBMS 82, Am. Math. Soc., Providence, Rhode Island (1993). 
[9] P. Schauenburg, Hopf-Galois and Bi-Galois Extensions, Fields Institute Communications 43 (2004), 469-515.

[10] P. Schaunnburg, Galois correspondence for Hopf Bigalois extensions, J. Algebra 201 (1990), 53-70.

[11] P. Schauenburg, Galois objects over generalized Drinfeld doubles, with an application to $u_{q}\left(s l_{2}\right)$, J. Algebra 217 (1999), 584-598.

[12] S. Skryabin, Projectivity and freeness over comodule algebras, Trans. Amer. Math. Soc. 359 (2007), 2597-2623.

Facultad de Matemática, Astronomía y Física, Universidad Nacional de Córdoba, Ciem - COniCET, (5000) Ciudad Universitaria, Córdoba, Argentina

E-mail address: galindo@mate.uncor.edu, natale@mate.uncor.edu 